\title{
Conséquences possibles de la libéralisation des échanges de sucre : Deux modèles et leurs réponses
}

Pré-print

\author{
Marie-Gabrielle Piketty (CIRAD-Ecopol), Jean Marc Boussard (INRA) \\ Contacts : piketty@,cirad.fr ; boussard@ivry.inra.fr \\ Article présenté au Colloque « Agriculture et commerce international », 6-7 février 2001 \\ INA-PG- Paris \\ Soumis à la revue Economie Rurale \\ Publié dans le numéro 270/juillet-août 2002
}

\section{Résumé}

Instabilité des prix et protectionnisme caractérisent le marché mondial du sucre. Après avoir souligné les spécificités de ce marché, les hypothèses, les résultats et les limites de deux modèles de la filière sucre mondiale sont analysés. La prise en compte de l'impact de l'instabilité des prix sur les décisions des agents peut modifier considérablement les résultats de la libéralisation des échanges de sucre, plaidant pour une meilleure représentation des fonctions d'offre dans les modèles utilisés au cours des négociations de l'OMC.

Titre en anglais : Can sugar market be liberalized ? Lessons from two models

\section{Summary}

Price volatility and protectionism are main features of the world sugar market. The assumptions, results and limits of two models of the world sugar market are analyzed. Taking account for the consequences of price volatility on agents decision making may radically change expected results of sugar trade liberalization, underlying the need to improve supply functions representation in models used during WTO negotiations.

Mots clés français : Sucre, Commerce International, modèle, risque, anticipations imparfaites

Mots Clés anglais : Sugar, International Trade, model, risk, imperfect forecast

JEL Classification : D46, D78, D84, F17, N50, Q1, R1 
Même s'il se voit parfois concurrencer par les sirops d'amidon ${ }^{1}$ (isoglucose, dextrose...) et les édulcorants intenses (saccharine, aspartame, traumatine...), le sucre reste de loin encore aujourd'hui le premier édulcorant, en assurant $86 \%$ de la production mondiale (Claus, 1998). Elément primordial de l'alimentation humaine, le sucre présente deux caractéristiques remarquables à l'aube des nouvelles négociations de l'OMC : son prix est des plus instables et il est jusqu'à maintenant passé au travers de la plupart des réformes des politiques agricoles, en particulier aux Etats Unis et en Europe, au grand regret des membres du groupe de Cairns, plus particulièrement l' Australie.

Après avoir présenté plus en détail les spécificités de ce marché et les liens possibles, mais équivoques, entre l'instabilité des prix mondiaux et la protection des producteurs de sucre (section I), les résultats et les limites de deux modèles supposés représenter le fonctionnement des marchés sucriers seront analysés et comparés. Ces modèles ont tous deux été construit dans le but d'évaluer les effets sur les prix des évolutions de la structure des échanges mondiaux de sucre et des politiques des différents pays. Il s'agit du modèle $S U G A B A R E$, utilisé par la délégation australienne à Seattle pour promouvoir la libéralisation des échanges (section II) et d'un modèle alternatif, développé depuis peu en France, qui cherche à rendre compte plus spécifiquement des conséquences de la volatilité des prix sur le comportement des agents (section III).

\section{I - Les spécificités du sucre et leurs conséquences sur le fonctionnement des marchés}

\section{I.1. Panorama général du marché}

La production de sucre peut venir de deux cultures : la betterave, produite dans les pays tempérés, et la canne, produite dans les régions tropicales. Cette dernière assure maintenant plus des $2 / 3$ de la production mondiale de sucre. La betterave permet d'obtenir directement du sucre blanc propre à la consommation alors que l'extraction de la canne débouche sur un produit brut (sucre « roux») qui doit être raffiné. Il y a donc deux marchés mondiaux du sucre, celui du roux et celui du blanc. La moitié des importations de sucre ces dernières années était sous forme de roux cependant la consommation finale est sous forme quasi-exclusivement de blanc.

Sur plus de 120 pays producteurs, huit pays totalisent plus de $60 \%$ de la production mondiale pour l'année 1999/2000 : le Brésil (19,2 millions de tonnes (MT)), les membres de l'Union Européenne (18,7 MT), l'Inde (18,4 MT), la Chine (9.1 MT), les Etats Unis (8 MT), la Thaïlande (5,8 MT), l'Australie (5,3 MT), et le Mexique (5,2 MT) (Chalmin 2000). La production de l'URSS et de Cuba a considérablement diminué à partir des années 1980 ; les pays protégés (pays de l'Union Europénne et Etats Unis) ont connu une croissance modérée de leur production; les pays "libéraux" ont connu une croissance plus rapide de leur production (Claus, 1998).

Le panorama ne serait pas complet sans mentionner une «spécificité » brésilienne: $60 \%$ de la canne à sucre récoltée au Brésil était encore en 1997 destinée à la production

\footnotetext{
${ }^{1}$ Les sirops d'amidon s'avèrent un concurrent important surtout pour la consommation industrielle mais plus limité pour la demande finale, du fait de leur présentation (forme liquide).
} 
d'alcool dans le cadre du Programme Proalcool ${ }^{2}$. Le maintien de ce dernier est remis en cause et aujourd'hui pratiquement aucun nouveau véhicule «à l'alcool» n'est immatriculé contre plus de $90 \%$ il y a quinze ans. Le potentiel productif sucrier peut se trouver radicalement modifié par un changement de politique dans ce domaine : la chute des prix durant l'année 1999-2000 doit beaucoup à la réduction des débouchés dans les distilleries, provoquant une explosion de la production de sucre du Brésil depuis 1997 (Chalmin 2000).

Les échanges mondiaux de sucre ont considérablement augmenté depuis le début des années 1980 avec une croissance des exportations de 33\% entre 1980 et 1997 (Sheales et al., 1999). Les exportations mondiales s'élèvent à 36,7 millions de tonnes en 2000 (Chalmin 2000). Les principaux exportateurs de sucre sont le Brésil, Cuba, l'Union Européenne, l'Australie et la Thaïlande. On peut noter la croissance des exportations du Brésil, de l'Afrique du Sud et du Mexique ces dernières années alors que les exportations de Cuba et de la Thaïlande tendent à baisser. Presque la moitié des exportations de sucre blanc est assurée par le Brésil et l'Union Européenne. La géographie des échanges est assez marquée : le Brésil et Cuba sont les principaux fournisseurs du bassin Atlantique alors que l'Australie et la Thaïlande satisfont la majeure partie de la demande du bassin Pacifique (Sheales et al., 1999)

La plus grande partie des importations de sucre roux vient d'un petit nombre de grands producteurs : l'Union Européenne, la Russie, les USA, le Japon, la Corée du Sud et la Malaisie (60\% des importations totales). Les importations de sucre blanc sont réparties de manière plus uniforme entre différents pays, traduisant chez ceux-ci l'absence d'une capacité de raffinage.

\section{I.2 Instabilité des prix : cause et/ou conséquence des politiques protectionnistes?}

Le prix mondial du sucre est très volatil (cf Figure 1). Son histoire est jalonnée de flambées de prix suivies de périodes d'effondrement. Les flambées de prix ont été particulièrement spectaculaires entre le milieu des années 1950 et le milieu des années 1980 (Delmas et Guillemin, 1983). Le prix du sucre a ainsi atteint un record de 65 cts/livre en 1974 sur le marché de New York; à l'inverse en juin-juillet 1985, le prix du sucre a diminué jusqu'à 2,5 cts/livre sur le même marché (du Genestoux, 1998).

Un indice d'instabilité calculé sur la période 1980-1989 indique ainsi une valeur de $72,7 \%$ pour le sucre qui est bien supérieure à tous les indices des autres biens agricoles et alimentaires, lesquels sont compris entre 7,9\% et 34,6\% (UNCTAD Commodity Yearbook cité dans Hannah et Spence, 1996).

\footnotetext{
${ }^{2}$ Programme mis en place au moment des chocs pétroliers par des politiques brésiliennes très interventionnistes afin de réduire la dépendance du pays par rapport aux fluctuations du prix du pétrole.
} 
Figure 1 : Prix mondiaux mensuels du sucre blanc $\left(n^{\circ} 11\right) 1800$ - 2000

Source : globalfinddata.com

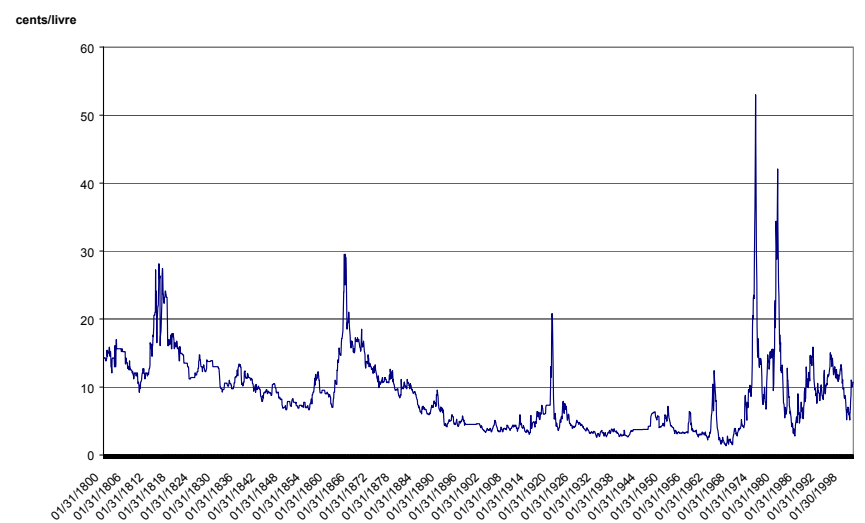

Les raisons les plus souvent invoquées pour expliquer ces très fortes volatilités des prix peuvent se synthétiser ainsi :

(i) L'ajustement de la production à la demande est lente : le sucre est à la fois un produit agricole et un produit industriel, qui demande un capital fixe élevé et la canne est une plante pluriannuelle qui continue notamment à être récoltée même quand les prix sont bas (du Genestoux, 1998 ; Hannah et Spence, 1996).

(ii) Le ratio échanges/production est faible et les échanges libres sont encore plus limités. $\mathrm{Du}$ fait des nombreuses politiques protectionnistes, ces derniers représentent seulement $17 \%$ de la production mondiale au milieu des années 1990 . Tout ajustement de l'offre ou de la demande dans les différents pays est alors amplifié par un marché mondial trop étroit (Delmas et Guillemin, 1983; Hannah et Spence, 1996; du Genestoux, 1998).

(iii) Avant 1990, les gouvernements assuraient une grande partie des échanges et faisaient des mauvais choix en terme de politiques (Wong et al, 1989 ; du Genestoux, 1998).

(iv) Les aléas climatiques perturbent les volumes de production (Delmas et Guillemin, 1983). Ceci est d'autant plus vrai que la canne est cultivée en région tropicale, où les aléas peuvent avoir des conséquences dramatiques (Hannah et Spence, 1996) ${ }^{3}$.

(v) L'incertitude de l'environnement conduit à des achats préventifs et à une spéculation inévitable (Delmas et Guillemin, 1983) ${ }^{4}$.

(vi) Jusqu'à la fin des années 1980, les importateurs étaient majoritairement des pays développés avec une élasticité faible de la demande par rapport au prix, ce qui a tendance à accroître la volatilité des prix. Depuis, les pays en développement sont responsables des deux tiers de la demande d'importations et cette demande, plus élastique par rapport au prix, tend à stabiliser les fluctuations du marché (Hannah et Spence, 1996).

\footnotetext{
${ }^{3}$ Comme le fait remarquer l'un des lecteurs, l'argument est à double tranchant : En concentrant la production dans les zones géographiques « les meilleurs », mais forcément de dimensions restreintes, l'exploitation maximale des avantages comparatifs rend la production globale plus sensible à des désastres météorologiques qui passeraient inaperçus avec un production plus uniformément répartie sur le globe.

${ }^{4}$ Un tel argument pourra surprendre : en principe, la spéculation est stabilisatrice. En réalité, la spéculation augmente les fluctuations quand les spéculateurs se trompent, une éventualité qui n'est pas envisagée dans les modèles néoclassiques à anticipations rationnelles, mais qu'il faut tout de même bien prendre en considération dans le monde réel.
} 
Jusqu'à récemment les prix semblent avoir connu moins de perturbations (cf Figure 1), ce qui pouvait conforter les arguments de certains (Hannah et Spence, 1996). Pour ces derniers auteurs, c'est l'accroissement de la demande d'importation des pays en développement (dont l'élasticité prix de la demande est plus élevée) et la dérégulation des marchés dans le sens d'une plus grande libéralisation, qui sont responsables d'une meilleure stabilité des prix sur les marchés mondiaux à partir de la fin des années 1980. Cependant la chute observée des prix en 1999 montre qu'il est encore bien tôt pour suggérer que cette stabilité est assurée.

Les fluctuations des prix du sucre ont des conséquences sur les politiques nationales. Depuis les années 1960, le degré d'auto-approvisionnement en sucre a augmenté dans les différents pays (Hannah et Spence, 1996). La nécessité d'une politique protectionniste est d'ordinaire justifiée par le souci de moins souffrir des aléas du marché : lors des flambées de prix, qui rendent les importations excessivement coûteuses, les pays tendent à investir dans la production, mais les entreprises sont ensuite mises en péril lorsque les prix mondiaux s'effondrent. Les pays exportateurs ont le même problème de gestion des revenus qui sont alors très variables sans protection. On peut souligner également qu'entre 1950 et 1994 (45 ans) 19 années ont été couvertes par des accords internationaux sur le sucre qui ont tenté sans succès de maintenir les fluctuations dans des bornes plus raisonnables (Hannah et Spence, 1996).

La plupart des pays intervenant sur le marché du sucre ont ainsi mis en place depuis fort longtemps des politiques de protection aux frontières et de régulation de l'offre. Avant les accords de Marrakech, celles-ci étaient constituées principalement de prélèvements variables sur les importations, de quotas à prix garantis et de restitutions à l'exportation. Depuis, les méthodes ont un peu varié, mais tendent toujours au même résultat, des prix intérieurs bien supérieurs au prix mondial et plus stables. L'Union Européenne, les Etats Unis et le Japon sont souvent montrés du doigt car les régulations y sont particulièrement fortes et pénaliseraient à l'extrême les consommateurs ${ }^{5}$. Mais la plupart des pays interviennent sur ce marché, même ceux qui se disent parfaitement libéraux comme l'Australie, le Brésil et la Thaïlande ${ }^{6}$.

La situation serait donc bloquée dans un cercle vicieux où l'instabilité des prix entraînerait des politiques protectionnistes qui elles-mêmes renforceraient l'instabilité alors qu'il suffirait d'élargir le marché par la libéralisation pour sortir de ce mauvais pas ? Nous allons voir que ceci dépend d'une hypothèse forte qui n'est pas toujours vérifiée.

\section{I.3. Que se passe-t-il lorsque la volatilité est une résultante endogène du fonctionnement des marchés?}

\footnotetext{
${ }^{5}$ D'où la thèse popularisée par les analyses célèbres, mais un peu anciennes de Olson (1987) ou Gardner (1992), selon laquelle les agriculteurs auraient, par leur pouvoir politique, et leur aptitude au lobbying, réussi à se faire octroyer des avantages exorbitants. Sans oublier leur importance, l'analyse de ces possibles « rentes de situation » n'est pas l'objet de cet article.

${ }^{6} \mathrm{Il}$ n'est pas lieu ici de décrire dans le détail ces politiques. On peut se référer pour le cas de l'Union Européenne en particulier à l'article de B. Bourges (1998) et pour l'ensemble des pays aux ouvrages de Hannah et Spence (1996), du FIRS (1997) et aux différents travaux du groupe ABARE (Wong et al 1989, Hafi et al.1993, Sheales et al. 1999).
} 
S'il est sûr que la volatilité des prix mondiaux du sucre explique le recours à des politiques visant à stabiliser les prix intérieurs, il faut revenir plus particulièrement sur l'argument (ii) qui consiste à dire que l'élargissement du marché mondial du sucre, que permettrait en fait la libéralisation des échanges, est de nature à diminuer les fluctuations des prix et, par voie de conséquence, améliorer grandement la situation actuelle, tout en diminuant les rentes de situation indues.

Les producteurs agricoles d'une manière générale, et les producteurs de sucre encore plus, compte tenu de l'instabilité des prix qui a été évoquée précédemment, ont peu de chance de connaître à l'avance le prix auquel ils vont vendre leur produit au moment de la récolte, ni même leur volume exacte de production, en raison des aléas climatiques. Par conséquent, les décisions sont prises sur la base d'un prix anticipé, qui peut tenir compte de toute l'information disponible au moment de la plantation mais il subsiste toujours une incertitude sur ce prix et sur les volumes finaux produits. Il devient alors nécessaire de faire intervenir dans les processus de décision des agents les erreurs d'anticipation et le risque.

Il existe, de ce point de vue, deux sortes bien distinctes de risques, trop souvent confondues dans la théorie économique contemporaine. Ce sont, d'un côté, le risque « exogène », qui provient de circonstances entièrement externes au système économique, comme, par exemple, la météorologie, et de l'autre, le risque "endogène », qui est, en quelque sorte, créé par le fonctionnement même du marché, et qui est au centre de la présente étude ${ }^{7}$.

Lorsque l'on considère que le risque pris par les producteurs est exogène, il induit certes des comportements de précaution, mais il garde une propriété remarquable qui est liée au fait qu'il ne se manifeste pas de la même manière dans les différentes régions de la planète. Dans ces conditions, l'élargissement des marchés est favorable au jeu de la loi des "grands nombres » : l'offre globale d'un produit agricole est pratiquement constante et ne souffre pas des aléas ponctuels qui se produisent dans telle ou telle région. Ceci est un argument fort pour l'élargissement des marchés qui réduit l'ampleur globale des risques comme cela a été montré par plusieurs auteurs (Bale et Lutz, 1979 ; Blandford, 1983 ; Tyers, 1990 ; Tyers et Anderson, 1992).

Ceci ne semble plus vrai dès lors que la génération du risque est liée au processus de décision de l'ensemble des producteurs et à leurs erreurs d'anticipation. Les séries de prix obtenues par l'équilibre entre l'offre et la demande en tenant compte des anticipations imparfaites et du risque peuvent devenir chaotiques et, en conséquence, impossibles à prévoir ${ }^{8}$ Dans ces conditions, le risque est engendré par le fonctionnement des marchés lui-même et un élargissement des marchés ne conduit pas nécessairement à la réduction de ces fluctuations (Boussard, 1996). Les paramètres pour lesquels on obtient des séries chaotiques sont difficiles à déterminer. Il existe cependant des raisons de penser qu'une demande rigide et une offre

\footnotetext{
${ }^{7}$ Bien évidemment, cette distinction entre risque « exogène » et « endogène » est différente de celle, classique en théorie des assurances, entre risque « systémique » et « non systémique ». Un risque systémique est un risque non assurable en raison de sa dimension, qui n'est pas « petite» comme le veut la « loi des grands nombres ». En ce sens, un risque de prix est un risque systémique, parce que tous les assurés vont le subir simultanément, et, de ce fait, un risque endogène a beaucoup de chances d'être systémique. Mais la chute d'une comète sur la terre est aussi un risque systémique. Or celui ci n'a rien d'endogène : il n'est pas produit par le fonctionnement de l'économie.

${ }^{8}$ Cf Gérard, (1991), Chavas et Holt , (1993), et bien d'autres . Une introduction particulièrement claire aux mathématiques chaotiques se trouve chez Alligood et al. (1990)
} 
sensible au prix constituent des conditions propices à l'enclenchement d'une dynamique chaotique ${ }^{9}$.

Lorsque les marchés sont chaotiques, les prix fluctuent constamment entre certaines limites. Les producteurs et les commerçants à tous les niveaux prennent des primes de risque, qui apparaissent dans les comptabilités comme des profits, mais que l'on aurait tort de confondre avec des rentes de monopole, ce qu'elles ne sont pas. Les prix ne sont jamais égaux au coût marginal, comme ils le devraient dans la théorie économique simplifiée encore trop populaire aujourd'hui, et les pertes sociales sont celles qui on été étudiées dès le début des années 40 par de nombreux analystes aujourd'hui oubliés (Waugh, 1944) ${ }^{10}$.

Toujours est-il que ces résultats relativisent les idées courantes sur les avantages supposés de la libéralisation du marché du sucre pour en atténuer les fluctuations, et plus généralement des marchés agricoles. Pour aller plus loin et compte tenu de la sensibilité des résultats à des paramètres clefs, il est indispensable de tenter une expérience appliquée et c'est ce qui a été réalisé dans le cas du sucre (cf section III). Auparavant, il faut revenir sur le modèle ABARE, pour le présenter plus complètement.

\section{Le modèle SUGABARE et le démantèlement des politiques sucrières}

L’Uruguay Round n'a que peu réussi à modifier les politiques nationales sucrières si ce n'est que les prélèvements variables sur les importations ont été remplacés par des tarifs. $\mathrm{Au}$ sein de l'OMC c'est bien évidemment le groupe de Cairns qui se sert des différents arguments explicités dans la section I.2, pour que les réglementations qui encadrent le marché du sucre soient démantelées. Plus particulièrement, l'Australie a mis en place un outil dont elle se sert depuis maintenant plus de 10 ans, le modèle $S U G A B A R E$, pour quantifier les effets des évolutions de la structure des échanges mondiaux de sucre et des politiques des différents pays sur les prix. Plus récemment, ce modèle a été utilisé lors d'une conférence de Presse à Seattle pour promouvoir la libéralisation des échanges (Sheales et al., 1999) ${ }^{11}$.

\section{II.1. Les principaux résultats du modèle SUGABARE.}

Les postulats théoriques sont qu'un environnement plus libéral permettrait de déplacer la production des pays où elle est subventionnée et où les coûts sont élevés vers les pays où les coûts sont moins élevés. Il s'agit là d'une application directe de la théorie élémentaire des avantages comparatifs : du fait des barrières à l'échange, les ressources sont mal utilisées et les consommateurs paient leur sucre trop cher. La libéralisation leur permettrait de disposer de

\footnotetext{
${ }^{9}$ Une étude fine sur les sources de l'instabilité des cours de l'huile de palme montre également que l'accroissement des échanges ne conduit pas nécessairement à une diminution de cette instabilité (Voituriez 2000).

${ }^{10}$ Un des lecteurs de cette revue nous a fait remarquer que Waugh n'était pas du tout oublié aux USA, où de nombreux bâtiments universitaires portent son nom. C'est exact. Pourtant, si sa mémoire est honorée, ses enseignements sont loin d'avoir été conservés, comme il arrive à de nombreux grands économistes.

${ }^{11} \mathrm{C}$ 'est la raison pour laquelle nous mettons ici l'accent sur ce modèle là plutôt qu'un autre. Il existe, en effet, de nombreux modèles de la filière sucre. Williams et Isham (1999) en recensent dix-neufs, et leur inventaire n'est pas complet. Mais le modèle SUGABARE a ceci de particulier qu'il est mis en vedette dans les négociations internationales. Il revêt, par conséquent, une importance politique particulière. Nous n'en donnerons pas ici une description détaillée, qui n'est pas l'objectif de cet article. On la trouvera dans les références que nous donnons. Nous nous contenterons d'un « modèle du modèle », qui fait ressortir les points de discussion cruciaux.
} 
plus de revenus, et les "ressources libérées » par le passage à une production plus efficace seront mieux utilisées dans d'autres secteurs de production. En outre, l'élimination de ces politiques favoriserait une meilleure transparence des marchés et une meilleure planification de la production par les acteurs de la filière (anticipations moins fausses).

Dans la dernière version du modèle, il est acquis que l'Australie, la Thaïlande et le Brésil ont des politiques totalement libérales contrairement aux Etats-Unis, à l'Union Européenne et au Japon. Les simulations réalisées pour la Conférence de Presse à Seattle envisagent l'abandon des politiques protectionnistes individuellement dans chacune de ces trois dernières régions puis une libéralisation multilatérale des marchés, en supposant deux scénarios distincts pour la politique brésilienne (plus ou moins de sucre destiné à la production d'alcool) (Sheales et al., 1999). Les résultats principaux sont les suivants :

- Chacun des pays a individuellement intérêt à libéraliser son secteur sucre même si les autres ne font rien. L'ouverture du secteur permet en effet d'augmenter le prix mondial mais de baisser le prix intérieur dans ces différents pays. Les consommateurs de ces pays y gagnent et leurs gains sont supérieurs aux pertes enregistrées par les producteurs.

- Une libéralisation multilatérale aurait des effets encore plus bénéfiques. Elle entraînerait une augmentation de $41 \%$ du prix mondial (28\% si l'on suppose que les Brésiliens substituent une partie de leur production d'alcool par du sucre). Cette augmentation signifie quand même une amélioration du surplus des consommateurs dans les pays très protégés (baisse du prix intérieur). On ne calcule pas la baisse de surplus dans les pays directement soumis au prix mondial mais on suppose qu'ils parviennent à gagner des parts de marchés auparavant occupées par les pays protégés : globalement leur situation doit donc s'améliorer. Il n'y pas de calcul très précis finalement des gains et des pertes des uns et des autres dans ce dernier scénario.

- Les résultats sont sensibles aux hypothèses sur les choix du Brésil en ce qui concerne le programme Proalcool et le prix mondial baisse rapidement dès lors que l'on augmente la part de la production de canne brésilienne destinée au sucre.

La publication présentant ces résultats reste peu précise sur les hypothèses et la structure du modèle et sur la manière dont sont réalisées les simulations. Elle renvoie aux travaux précédents dont l'analyse est très intéressante (cf section II.2). On peut noter à ce titre qu'en 1989, les auteurs de ce modèle étaient beaucoup plus précis sur ses limites, et beaucoup plus prudents sur les résultats ${ }^{12}$.

\section{II.2. La structure du modèle et ses limites}

Le modèle construit par ABARE est constitué d'un système d'équations récursives, dans leur principe très semblables au modèle présenté dans la section suivante. Les agents sont d'abord les agriculteurs/producteurs, d'un côté, les consommateurs finaux, de l'autre.

\footnotetext{
12 En effet, en 1989, la politique sucrière australienne était encore plus interventionniste qu'en Europe. La conclusion principale de leurs simulations était alors qu'une petite augmentation de la part de la consommation et de la production qui répondrait directement au prix mondial (comme le cas de la production hors quota en Europe, qui est même montré en exemple), permettrait de diminuer les fluctuations mondiales des prix (Wong et al 1989). Ces résultats ont sans doute influencé le retrait du contrôle des surfaces allouées à la canne en Australie.
} 
Entre les deux, on trouve les raffineurs/stockeurs, ainsi que les exportateurs/importateurs. Chacun des agents est caractérisé par une équation de comportement (ou un ensemble d'équations, lorsqu'il faut par exemple distinguer le comportement des exportateurs vis à vis de l'Union Européenne, et vis-à-vis d'autres destinations). Le modèle est fermé par la nécessité d'équilibrer tous les marchés.

Comme cela a été la règle en économétrie pendant quelques décennies, les équations de comportement sont définies par des formes fonctionnelles plus ou moins arbitraires (par exemple «Cobb Douglas »), sans que ce caractère arbitraire soit jugé trop grave du fait de leur "flexibilité »: les fonctions en question dépendent de nombreux paramètres, que l'on peut ajuster pour les faire «coller » à presque toutes les situations possibles. De ce fait, on compte sur l'estimation statistique des paramètres inconnus pour produire des relations valables "en moyenne", "au voisinage" des conditions dans lesquelles elles ont été établies. C'est du reste ce qui fait une grande partie de sa force de persuasion, un grand nombre de gens croyant que l'utilisation de procédés d'estimation sophistiqués est une garantie de crédibilité. Il n'en est rien ${ }^{13}$, comme on va le voir plus loin.

Les propriétés dynamiques du système sont induites par les liaisons inter temporelles qui existent d'abord entre les stocks et les flux - il s'agit là, en général d'identités incontournables - et aussi entre l'offre et les prix passés. L'une de ces équations est celle qui détermine les conditions de stockage, et dans laquelle le décalage ne dépasse pas une année. L'équation la plus importante du modèle ${ }^{14}$, cependant, est l'équation de comportement des producteurs (Hafi et al., 1993):

(1) $Q_{i t}=F_{i}\left(P_{t-1}, . ., P_{t-k}, \prod_{i t-1}, \ldots \Pi_{i t-k}, Q_{i t-1, t}\right)$, où :

$\mathrm{F}_{\mathrm{i}} \quad$ est une fonction linéaire en logarithme, dont les coefficients font l'objet d'une estimation économétrique pour chaque pays.

$Q_{i t} \quad$ est la quantité de sucre produite l'année t dans le pays i

$P_{t} \quad$ est le prix mondial ${ }^{15}$ du sucre blanc l'année t,

$\Pi_{i t}$ est un indice des prix des cultures concurrentes du sucre dans le pays i l'année t

$t \quad$ représente un indice de temps discret annuel

$i$ représente un pays.

$k \quad$ représente la durée de temps pendant laquelle les prix passés sont supposés avoir de l'influence sur les décisions courantes, en l'espèce 4 ans.

C'est cette équation (avec un pas de temps annuel, nous reviendrons sur ce point) qui, confrontée à une demande rigide, engendre toute la dynamique complexe du modèle. Elle peut être interprétée comme la forme réduite d'un système de deux équations, à savoir:

\footnotetext{
${ }^{13}$ Le même type d'approche a été utilisé par exemple dans un modèle de l'agriculture française préparé à l'INRA dans les années 70 (Boussard, 1975), lequel n'a jamais donné de très bons résultats en terme de capacité prédictive, et c'est l'une des raisons pour lesquelles nous pouvons ici être catégoriques dans cette affirmation.

${ }^{14}$ Les autres équations du modèle n'ont pas de décalages dans le temps de rang supérieurs à l'unité, et, en outre ces décalages sont imposés par le bon sens (par exemple, le stock de l'année t est évidemment égal au stock de l'année t-1, plus les entrées, moins les sorties...).

${ }^{15}$ Il peut paraître surprenant que le "prix mondial du sucre " soit ici pris comme référence, au lieu du prix intérieur. Cela tient d'abord à ce que les coefficients des équations convertissent implicitement le prix mondial en prix intérieur au taux de change moyen sur la période d'estimation du modèle, ensuite à ce que, dans les pays à quotas, comme les membres de l'Union Européenne, c'est la production « hors quota » qui est prise en compte.
} 
- une "condition du premier ordre pour la maximisation du profit des producteurs ", qui fait dépendre la production des prix , et,

- une équation spécifiant la formation des anticipations, les prix anticipés, aussi bien du sucre que des cultures concurrentes, se trouvant être des moyennes pondérées des prix passés sur la durée $\mathrm{k}$.

Vue comme cela, cette équation est fortement ancrée dans la théorie économique standard, et reflète l'analyse courante selon laquelle la production est influencée par le niveau moyen des prix observés au cours d'un passé pas trop lointain, ce qui est indiscutable ${ }^{16}$. On peut cependant se demander si le niveau moyen des prix du sucre (et des prix des cultures concurrentes) est vraiment non seulement la seule variable susceptible d'agir sur les décisions des producteurs (il est clair que d'autres variables peuvent intervenir, mais elles sont prises en compte dans les élasticités qui expriment l'ampleur de la réaction moyenne des producteurs à une variation du prix moyen), mais encore si c'est bien la seule information transmise par le marché à ces derniers.

En réalité, le marché, en faisant varier les prix, donne des informations aussi bien sur la dispersion (ou la volatilité) des prix que sur leur niveau moyen. Dès lors, il peut délivrer des messages beaucoup plus ambigus que ceux qui sont véhiculés par les moyennes. Si le prix passe de 1 à 0,8 puis à 2 , cela veut il dire que la production devient cette année plus rentable, ou seulement plus dangereuse ? Selon la réponse apportée à cette question, l'effet de l'évènement sur un producteur peut être très différent. C'est pour cette raison que la spécification de fonctions d'offre comme celles qui figurent dans le modèle SUGABARE pose des problèmes redoutables quand il s'agit d'estimer les équations par des méthodes statistiques $^{17}$, et c'est ce qui fait que, de l'aveu même des auteurs, les paramètres n'en sont pas déterminés avec une très grande précision. Ces problèmes ne font que refléter la difficulté de donner à ce type d'équation une interprétation économique plausible.

De fait, l'hypothèse selon laquelle il serait possible de remplacer les grandeurs incertaines par leur moyenne dans des équations censées refléter les comportements des producteurs - en particulier des producteurs agricoles, gens plutôt pauvres dans de nombreuses régions du monde ${ }^{18}$ et donc en général assez prudents - ne tient absolument pas compte de tout ce que l'on sait sur l'influence du risque dans les décisions des agents économiques. On sait que les producteurs - agricoles en particulier - sont en général prêts à sacrifier de fortes espérances de gains contre une plus grande sécurité, et que cela dicte leurs décisions au moins autant que les espérances de rentabilité qu'ils peuvent avoir vis à vis de telle ou telle spéculation. Pour cette raison, les programmes de stabilisation de prix et autres assurances récoltes sont de nature à modifier considérablement leurs attitudes et leurs décisions. Il est donc impossible de considérer que les coefficients des équations estimées dans les modules de production du modèle SUGABARE seront les mêmes dans un régime de

\footnotetext{
${ }^{16}$ Naturellement, même en adhérant à cette hypothèse, le problème se pose de quelles pondérations adopter pour calculer la moyenne - et quelle formule de moyenne adopter . Ici, la pondération de la moyenne arithmétique est fournie directement par l'estimation statistique, ce que les spécialistes des séries chronologiques (Cf par exemple, Tong,1999) trouveraient sûrement discutable. On aurait pu songer à des techniques plus sophistiquées comme les « polynômes de Almond» (Almond, 1965). Les auteurs de ABARE ne l'ont pas fait.

${ }^{17}$ Voir, en particulier, parmi d'innombrables ouvrages traitant de la question, Nerlove, Grether et Carvalho (1995).

${ }^{18}$ En particulier, ceux des pays en voie de développement. Certaines catégories de producteurs de sucre, dans les pays de l'OCDE notamment, sont au contraire « relativement » riches.
} 
prix garantis, et dans un régime libéralisé, où l'ampleur des fluctuations de prix sera complètement différente ${ }^{19}$.

On voit ainsi que non seulement le modèle SUGABARE ne peut que difficilement prétendre analyser "ce qui se passerait si les prix étaient libéralisés", mais encore que, par construction, il s'interdit d'envisager l'étude des bénéfices ou des coûts de politiques destinées à agir moins sur les moyennes des prix que sur leur dispersion. Or, quand on envisage les politiques de libéralisation, c'est bien des politiques de ce genre que l'on veut évaluer, personne ne croyant sérieusement que les coûts de production du sucre, et donc les prix correspondants, ne puissent varier dans des proportions vraiment importantes ${ }^{20} \mathrm{du}$ seul fait du démantèlement des politiques actuelles.

C'est pourquoi, en dépit de l'existence du modèle australien construit avec une rigueur et un professionnalisme auquel nous rendons hommage, il nous est apparu nécessaire de développer un modèle alternatif, susceptible de tenir compte des problèmes qui viennent d'être évoqués. Le modèle développé ici a pour caractéristique principale que chacune des équations de comportement analogue à celles du modèle ABARE y comporte un volet "risque" qui lui donne des propriétés entièrement différentes.

Le prix à payer pour cela est que nous avons dû renoncer à l'estimation statistique des coefficients de nos équations. L'estimation statistique des paramètres de grands systèmes d'équations non linéaires n'est certes pas impossible, mais elle est délicate avec des séries chaotiques. De plus, nous pensons que la qualité des résultats d'un modèle dépend bien plus de la qualité et de la pertinence des équations que l'on y met que de la qualité des estimations économétriques de coefficients d'équations moins pertinentes. Enfin, nous nous sommes largement servis des estimations mêmes de ABARE chaque fois que cela a été possible (en particulier, celles qui concerne les élasticités de demande).

\section{Les conséquences de la prise en compte de la volatilité des prix, du risque et des erreurs d'anticipations}

\section{III.1 Quelques hypothèses du modèle proposé}

Le modèle présenté ici comporte plusieurs différences par rapport au modèle ABARE qui vient d'être décrit. Nous insisterons ici sur quelques unes des plus importantes en rapport avec le sujet précis de cet article ${ }^{21}$. Les hypothèses qui ont été faites se sont inspirées de plusieurs discussions avec les professionnels du secteur dont nous avons tenté de respecter les avis. On distingue six types d'agents dans chaque pays (le cas échéant bien sûr), les agriculteurs, les industriels du sucre, les stockeurs de sucre blanc et de sucre roux, les raffineurs et les consommateurs finaux. Un diagramme simplifié du modèle se trouve en annexe.

\footnotetext{
${ }^{19}$ William et Isham (1999) font la même constatation dans le cas du sucre : « quand on étudie des alternatives politiques « avec et sans stabilisation », il est clair qu'il faut introduire l'incertitude dans le modèle ».

${ }^{20}$ Des variations de quelques pourcents ou même de quelques dizaines de pourcents, sont peu de chose vis à vis des fluctuations présentes des cours dans un marché pourtant largement contrôlé et régulé.

${ }^{21}$ La description complète du modèle se trouve dans Boussard et Piketty (2001).
} 
Le pas de temps du modèle est mensuel, caractéristique importante du fonctionnement des marchés, afin de respecter les calendriers de production dans les différents pays et raffiner le traitement des anticipations des différents acteurs. Ainsi, les agriculteurs plantent à une date précise dans l'année et fixent les termes de leur contrat avec les sucreries en fonction de l'information disponible à cette date, sur la base de moyennes et de variances annuelles (cf infra). La récolte est répartie dans chaque pays à des dates précises, donne lieu à une transformation en sucre et va alimenter mécaniquement les stocks à cette période. Par conséquent, l'offre ne s'ajuste pas instantanément à la demande : une fois que la décision de plantation est prise, l'offre des agriculteurs, qui ne sera disponible sur le marché que plusieurs mois après, est fixée. Les décisions de stockage et de déstockage sur le marché mondial par contre sont prises chaque mois en fonction d'anticipations mensuelles. Dans ce deuxième type de décision, conformément aux dires d'experts, on a pris en compte l'influence de l'état des stocks sur les anticipations mensuelles ${ }^{22}$. Chaque mois, le prix mondial du sucre roux équilibre les décisions de ventes des stockeurs de roux et la demande des raffineurs, tandis que le prix mondial du sucre blanc équilibre les ventes des stockeurs de sucre blanc avec la demande des consommateurs finaux. Enfin, les capacités de production des sucreries et des raffineurs peuvent être modifiées une fois par an en fonction des résultats obtenus les années précédentes et des anticipations des industriels.

Les séries mensuelles de prix obtenues par le modèle peuvent être caractérisées par un niveau moyen et par une dispersion par rapport à ce niveau moyen, que l'on peut mesurer par sa variance. A tous les échelons de décisions (sauf pour la consommation) on suppose que la moyenne et la variance influencent le choix des agents. On utilise pour cela une formulation classique de type espérance variance : en considérant U, la fonction d'utilité de l'agent considéré (souvent le profit moins les coûts pour le producteur), au lieu de considérer simplement et classiquement que l'agent maximise cette fonction en tenant compte de toute une série de contraintes, on considère qu'il maximise une fonction de type $E(U)-1 / 2 A^{*}$ Var $(\mathrm{U})$, avec $\mathrm{E}(\mathrm{U})$ l'espérance de la fonction d'utilité, $\operatorname{Var}(\mathrm{U})$ la variance de l'utilité et $\mathrm{A}$ un coefficient d'aversion pour le risque (Markowitz, 1970).

La modification fondamentale que cela entraîne, par exemple dans le cas de la production de sucre dans chaque pays, est qu'elle dépend maintenant non seulement du niveau des prix mais aussi de la volatilité observée de ces prix au cours des périodes précédentes. On aura une fonction du type

$$
Q_{i t}=F\left(\overline{P_{i, t}}, A_{i} * \sigma_{i, t}^{2}, C_{i}\right)
$$

avec

$\mathrm{t} \quad$ mois où se prend la décision de production

$\mathrm{Q}_{\mathrm{i}, \mathrm{t}} \quad$ le volume de produit décidé à la date $\mathrm{t}$

$\overline{P_{i, t}} \quad$ l'espérance du prix du sucre observée à $\mathrm{t}$ (qui dépend des prix observés les périodes précédentes comme dans l'équation 1)

$\mathrm{A}_{\mathrm{i}} \quad$ l'aversion pour le risque du producteur

$\Phi^{2}{ }_{i, t} \quad$ la variance du prix observée à la date t qui dépend des écarts à la moyenne des prix observés durant les périodes précédentes

\footnotetext{
${ }^{22}$ Ceci est apparu au cours de plusieurs discussions. En outre, Delmas et Guillemin (1983) avancent que les prix mondiaux seraient ainsi déprimés lorsque les stocks excèdent 5 mois de consommation et des hausses records apparaîtraient lorsque les stocks atteignent un niveau plancher équivalent à trois mois de consommation. Cette relation simple ne se vérifie cependant pas systématiquement ainsi, la flambée des prix de 1980 serait vraisemblablement due à l'anticipation erronée d'une future pénurie.
} 
$\mathrm{C}_{\mathrm{i}} \quad$ les coûts de production pour produire une quantité $\mathrm{Q}_{\mathrm{i}, \mathrm{t}}$

Plus précisément, alors que le volume de production est une fonction croissante de l'espérance du prix observée, il est une fonction décroissante de la variance. En outre plus la production est importante plus le poids de la variance est fort. C'est là une différence considérable par rapport à la situation standard étudiée par la théorie économique élémentaire, dans laquelle seul le niveau moyen des prix provoque des réactions des producteurs. Ici un accroissement de la variabilité produit une baisse de la production et par réaction une augmentation du prix mondial. Ceci différencie fondamentalement une production sous quotas à un prix garanti, comme les quotas $\mathrm{A}$ et $\mathrm{B}$ en Europe par exemple et une production sur un marché libre. Dans le premier cas, l'offre est simplement croissante en fonction du prix du quota dans les limites du quota et le prix mondial influence la décision des producteurs uniquement pour la production hors quotas. Dans les pays où il existe des taxes fixées sur les importations, l'espérance du prix observée tient compte de cette taxe ; lorsque le ratio de cette taxe sur le prix mondial est fixé (taxe ad-valorem), la variabilité des prix mondiaux influence toujours les producteurs.

De la même manière ce type de formulation se retrouve au niveau du stockage : les agents arbitrent chaque mois entre garder une certaine quantité en stocks en espérant les vendre plus cher le mois prochain à un prix anticipé et vendre une certaine quantité actuellement au prix d'équilibre du marché. Plus la variance des prix est élevée, plus les agents tendent à vendre immédiatement. Ici encore, tout changement de la volatilité va avoir des conséquences sur l'offre et sur le consommateur final. Pour le traitement particulier de l'espérance, on prend en compte le niveau des stocks mondiaux (cf supra) et la différence entre le prix observé le mois précédent et un prix de long terme de référence : les stockeurs anticipent une hausse du prix si le prix observé le mois précédent est inférieur au prix de long terme de référence et inversement dans le cas contraire ; ces anticipations sont corrigées par le niveau des stocks restant.

\section{III.1. Quelques résultats illustratifs}

Le monde a été divisé en seize grandes régions ou pays : 1'Australie, le Brésil, Cuba, l'Inde, la Thaïlande, l'Afrique du Sud, les pays d'Amérique Centrale, ACP, d'Afrique Subsaharienne, les anciens pays de l'Union Soviétique, les pays d'Europe de l'Est, l'Union Européenne, le Japon, les Etats Unis, les pays du Maghreb - Machrek et les autres pays asiatiques. Dans la situation de référence il existe deux grands types de politiques, que l'on a volontairement simplifiés dans cette première étape :

- des quotas à prix garantis en particulier pour l'Union Européenne, les Etats Unis, les pays ACP, l'Australie, le Brésil, l'Afrique du Sud, les pays d'Amérique Centrale, et les pays d'Afrique Sub-Saharienne ;

- des taxes sur les importations en particulier au sein de 1'Union Européenne, des anciens pays de l'Union Soviétique, en Europe de l'Est, aux Etats-Unis et au Japon.

Une première simulation de référence est réalisée en considérant que rien ne bouge en terme de politiques économiques. Le taux de croissance de la population est fixé de manière exogène dans chaque région. Un module d'investissement permet une fois par an, dans chaque pays, d'actualiser les capacités de production et de raffinage en fonction des résultats 
obtenus les années précédentes (Boussard et Piketty, 2001). Puis, dans un deuxième scénario, on supprime toutes les interventions, le marché est parfaitement libre ${ }^{23}$.

La Figure 2 représente les séries de prix obtenues dans le scénario de référence sur 20 ans. Bien sûr tout modèle de ce type se doit de passer un test de validation. La difficulté de l'exercice dans le cas présent tient au fait que les séries obtenues sont chaotiques ${ }^{24}$. Avec les séries chaotiques, la capacité de prédiction ne constitue plus un bon critère de qualité, puisque, par hypothèse, une faible erreur «au départ» entraîne une erreur très grande au bout de quelques périodes. Il est tout de même possible de valider par comparaison avec la réalité un modèle qui prédit une série chaotique, si l'on songe que ce qui est prédit n'est pas une valeur de la série plutôt qu'une autre, mais seulement un régime de variabilité, une loi de probabilité, une «distribution ». Ceci conduit à focaliser l'attention sur l'allure des courbes obtenues au cours du temps.

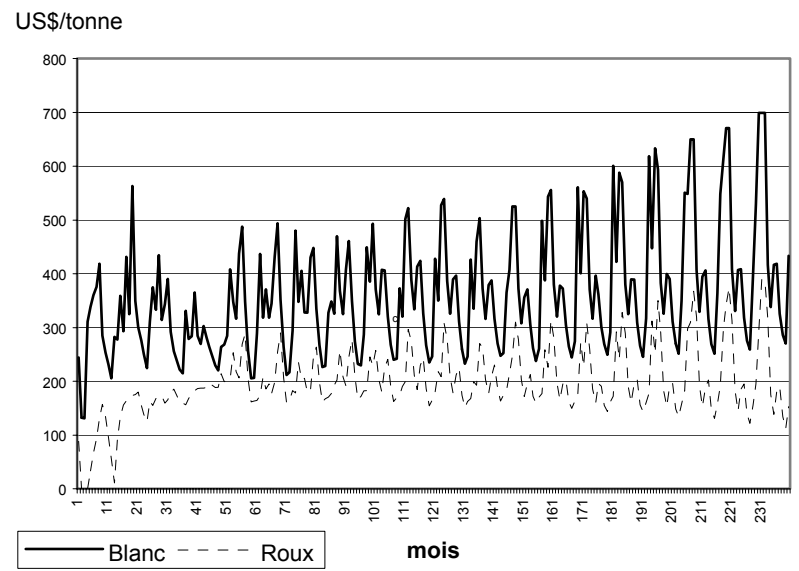

Figure 2 : Séries de prix dans le scénario de référence

La validation a été réalisée en comparant les 120 premières puissances spectrales des transformées de Fourier des séries obtenues avec celles d'une série réelle des prix du sucre. Cette comparaison montre que les séries présentent de grandes similarités, indiquant que le modèle, malgré ses imperfections, a réussi à saisir une large fraction de la variabilité de prix du sucre (Boussard et Piketty, 2000).

\footnotetext{
${ }^{23}$ Là encore, seuls quelques résultats seront présentés, l'ensemble pouvant être consulté dans Boussard et Piketty (2000).

${ }^{24}$ Le mot chaotique est ici pris dans le sens technique en mathématique qui implique en particulier, «sensibilité aux conditions initiales », « non linéarité » et « corrélation de tous les moments »
} 
Figure 3 Prix mensuel du sucre blanc avant et après la libéralisation

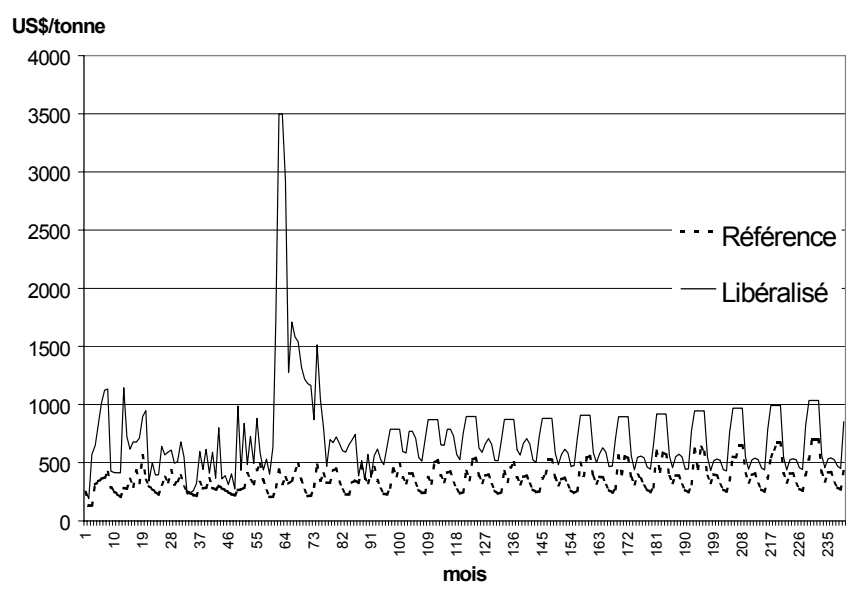

Lorsque l'on passe au scénario libéral, on retrouve, au niveau de l'évolution globale des prix, des résultats similaires à ceux du modèle $S U G A B A R E$ : les prix augmentent. Cependant, ils augmentent beaucoup plus et surtout ils deviennent beaucoup plus volatiles (cf Figure 3).

Cette hausse des prix est assez importante et pénalise les consommateurs dans la plupart des régions du monde (cf Figure 4). En calculant la variation du surplus du consommateur ${ }^{25}$ entre le deuxième et le premier scénario, on constate que seuls les consommateurs japonais et européens voient leur situation s'améliorer. Par rapport aux résultats de $S U G A B A R E$, la hausse plus importante des prix fait que même certains pays comme les pays de l'ex-URSS, d'Europe de l'Est et les Etats-Unis qui ont des taxes à l'importation élevées dans la situation de référence, paient un prix supérieur avec un marché libre.

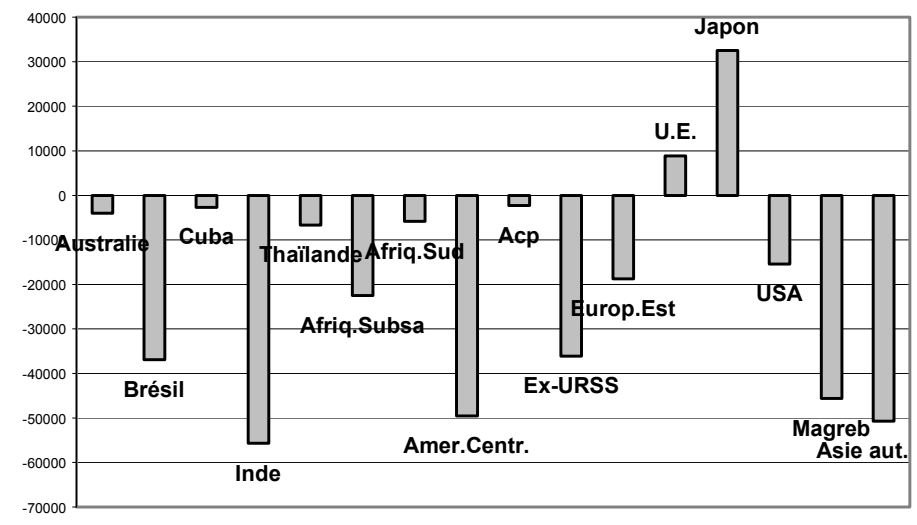

Figure 4 : Evolution du surplus cumulé des consommateurs avec le libre -échange

La hausse des prix peut laisser croire que les producteurs ont gagné beaucoup au libre échange. Cependant, ce n'est pas si simple: si en moyenne les prix augmentent, leur variabilité aussi. Par conséquent l'effet sur la production n'est pas toujours positif. Ainsi,

\footnotetext{
${ }^{25}$ Le surplus du consommateur est ici calculé en utilisant la courbe de demande donnée $a$ priori (en fait, une exponentielle à élasticité constante) et en calculant simplement la variation de l'intégrale entre les deux prix à comparer chaque mois. Puis on effectue la somme algébrique de ces surplus mensuels. Nous sommes évidemment conscients des limites de cet indicateur, maintes fois critiqué, et à très juste titre. Nous le faisons apparaître ici parce qu'il est habituellement utilisé dans les modèles qui évaluent les bénéfices et les coûts de la libéralisation des échanges.
} 
seuls le Brésil, l'Inde et l'Afrique du Sud arrivent à augmenter leur production de manière significative. Tous les autres pays enregistrent une baisse nette des volumes produits, même l'Australie et la Thaïlande (dans de faibles proportions bien sûr par rapport à d'autres régions comme l'Union Européenne et les pays de l'ex-URSS), ce qui est là encore bien différent des résultats du modèle australien. Par conséquent, l'effet de l'augmentation des prix sur les revenus des producteurs dans les différents pays n'est pas toujours positif (Boussard et Piketty, 2000) ${ }^{26}$.

Finalement, il faut ajouter que parmi les différents acteurs de la filière sucre, les raffineurs seraient les grands gagnants de la libéralisation, notamment dans les pays développés (Boussard et Piketty, 2000). Ceci est lié au fait que l'écart se creuse entre les prix du roux et les prix du blanc dans le second scénario, au profit du sucre blanc. Cette conclusion recoupe celle de Williams et Isham (1999), qui l'attribuent à la rigidité de l'appareil de production des raffineurs, qui joue aussi dans notre cas. Mais il est clair que cette rigidité existe aussi dans les systèmes " protégés ", de sorte qu'il faut admettre que cette rigidité est particulièrement dommageable dans un marché fluctuant.

\section{III.2. Limites et améliorations possibles}

La principale limite de cette seconde approche est liée à la faiblesse des données empiriques sur lesquelles le modèle repose pour le moment. C'est pourquoi, on ne peut donner aujourd'hui que des résultats illustratifs et il n'est pas encore possible d'utiliser sans danger cet outil dans les négociations internationales. Ceci est facilement remédiable pour certains aspects mais plus difficile, car novateur pour d'autres par rapport aux estimations classiques de fonctions d'offre, de déstockage etc... : il s'agit en particulier de l'estimation du poids donné à la variabilité des prix dans les choix. Par exemple, dans le scénario présenté ici, l'effondrement de la production dans certains pays est liée à un poids particulier donné à la variance des prix de manière $a d-h o c$ et il est responsable de la hausse plus rapide des prix. Il n'en reste pas moins que c'est une lacune à combler et qu'il n'est pas plus juste, compte tenu de ce qui a été vu dans la première section, d'ignorer complètement et sans fondements l'influence de la variabilité des prix sur les décisions des agents.

Par ailleurs, le modèle est très sensible au mode d'anticipations que l'on fixe et il ne converge plus parfois après plusieurs périodes pour certaines valeurs de paramètres. Toute la difficulté réside dans la manière de représenter ces anticipations et leur évolution au cours du temps. Il faut souligner à ce titre une limite visible dans le scénario libéral présenté ici, liée à la périodicité des prix après la neuvième année, phénomène qui ne pourrait se prolonger dans un monde réel $^{27}$. Ceci nécessite un meilleur traitement des anticipations, et ce sera évidemment l'objet de nos recherches futures.

Enfin, une omission importante de ce modèle est celle de l'existence des " marchés à terme » qui, en permettant aux producteurs de «s'assurer» contre les fluctuations de prix, sont de nature à relativiser l'importance que nous avons attribuée aux fluctuations de prix. De

\footnotetext{
${ }^{26} \mathrm{Il}$ faut ajouter que les revenus sont distribués entre les stockeurs, les industriels et les agriculteurs, ce à des dates différentes au cours de l'année, et en faisant intervenir des contrats entre les agriculteurs et les industriels. Par conséquent, les fluctuations des prix peuvent également avoir des effets redoutables en terme de répartition des revenus entre les différents acteurs de la filière.

${ }^{27}$ En effet, on ne peut ignorer dans ce cas que les agents seraient les premiers à percevoir ces cycles et à modifier leurs anticipations de manière à en tirer partie, modifiant par la même les nouveaux équilibres de prix des marchés.
} 
fait, ils sont souvent présentés comme «la » solution libérale aux problèmes causés par l'instabilité des prix, de même que l'assurance intercontinentale est la solution aux aléas météorologiques. Même s'il s'en faut de beaucoup, que ce parallèle soit complètement justifié c'est incontestablement une faiblesse du présent modèle que de ne pas en avoir tenu compte. Ici encore, nous espérons combler cette lacune dans l'avenir.

Il n'en demeure pas moins que, même avec ces omissions, nous ne connaissons aucun modèle de la filière sucre qui tienne compte d'une aussi vaste variété de mécanismes (qu'il s'agisse des anticipations, de la rigidité des structures de production, de la dynamique et du risque) que ceux qui ont été introduits ici. C'est pourquoi, tout en étant très prudents sur l'interprétation des résultats, nous pensons que ceux-ci gardent un certain degré de signification au moins qualitative.

\section{Conclusion}

Faut-il se fier à l'optimisme des Australiens qui avancent des gains importants si les marchés du sucre étaient libéralisés ? Faut-il rester dans la situation actuelle, certes imparfaite, au motif que la libéralisation des échanges, même si elle semble permettre une meilleure allocation des ressources, risque en même temps d'accroître la volatilité des prix du sucre, déjà élevée, et de mettre en péril la sécurité des approvisionnements mondiaux ? Aujourd'hui, les arguments et les outils disponibles ne permettent pas de trancher ou de proposer des alternatives, le cas échéant soit parce que les modèles ne représentent pas de manière juste le marché et ses différents acteurs soit parce qu'ils sont encore insuffisamment robustes sur le plan empirique. Il nous semble qu'améliorer la représentation du fonctionnement des marchés dans un modèle de la filière sucre mondial, comme cela a été commencé ici, est nécessaire pour avancer dans ce type de débat et y apporter de nouveaux éléments de discussion.

Parmi les conclusions importantes de cette contribution, il faut insister sur l'importance la prise en compte du risque « endogène » et des anticipations imparfaites dans les modèles qui cherchent à évaluer les bénéfices et les coûts de la libéralisation des échanges sur le marché du sucre. Nos résultats à ce titre ne sont pas en accord avec les conclusions de Williams et Isham (1999), qui discutant des mérites respectifs des différents modèles de la filière sucre, remarquent que « La valeur ajoutée par la prise en compte de l'incertitude année par année est faible par rapport à l'accroissement de complexité mathématique du modèle ». Ceci est lié au fait que ces auteurs n'envisagent comme source d'incertitude " annuelle », justement, que les fluctuations météorologiques, source de risque « exogène ».

\section{Références}

Alligood, K., T.D. Sauer, and J.A. Yorke (1997) : Chaos : An introduction to dynamical systems. Springer, New York.

Almond, S. (1965): The distributed lag between capital appropriation, and expenditures Econometrica 33:178196.

Bale, M. Lutz E. : The effect of trade intervention on international price instability. AJAE, 1979, 61 (3), pp. 512 -516 . 
Bourges, B. : L'organisation commune du marché du sucre dans l'Union Européenne et les échanges mondiaux. Comptes Rendus de l'Académie d'Agriculture de France, 1998, 84 (8), pp 31-64.

Blandford, D.: Instability in world grain markets. Journal of agricultural economics, 1983, 34, pp. 379-392.

Boussard, J.M. : La production agricole française: un modèle historico statistique INRA. Paris, 1975.

Boussard, J.M. : When risk generates chaos. Journal of economic behavior and organization, 1996, 29, pp.433446.

Boussard, J.M., M.G. Piketty : Can markets supports trade? The case of sugar. Paper presented to the Congress of the International Association of Agricultural Economist, Berlin August 2000.

Chalmin, P. : (ed.) Cyclope. Les marchés mondiaux. Paris, Economica, 2000.

Chavas J.P. et M.T. Holt (1993) : Market instability and non linear dynamics, AJAE 75 : 113-120.

Claus, R. : La production mondiale de sucre. Grandes masses et principaux producteurs. Comptes Rendus de l'Académie d'Agriculture de France, 1998, 84 (8), pp. 5-15.

Du Genestoux, P : Le marché mondial du sucre. Comptes Rendus de l'Académie d'Agriculture de France, 1998, 84 (8), pp 17-30.

FIRS : L'économie sucrière. Campagne 1995-1996. Perspectives 1997. FIRS, Paris, 1997.

Gardner, B.L. : Changing economic perspectives in the farm problem. Journal of Economic Literature 30 : 62101.

Gérard, F. : Instabilité des prix agricoles et influence de l'incertitude sur les comportements économiques : essai sur les problèmes associés à la régulation de l'offre. Thèse de Doctorat, Université de Paris I, 1991.

Hafi, A., P. Connell,and R. Sturgiss : Market potential for refined sugar exports from Australia. ABARE research report $N^{\circ}$ 93-17, Canberra, 1993.

Hannah, A.C., D. Spence : The International Sugar Trade. Woodhead Publishing Ltd., Cambridge, 1996, 245 p..

Markowitz, H.M.: Portfolio analysis : efficient diversification of investments. Yale University Press. Yale, 1970.

Nerlove, M, D.M. Grether and J. L. Carvalho : Analysis of Economic Time Series: A Synthesis. Academic press, San Diego, 1995.

Olson, M. : The logic of collective action. Traduction : Logique de l'action collective. PUF, Paris, 1987.

Sheales, T., S. Gordon, A Hafi, and C. Toyne : Sugar : Intervention policies affecting market expansion. ABARE Research Report N 99.14., Canberra, 1999.

Tong, H. (1999) Non linear time series . Oxford University press, Oxford.

Tyers, K : Implicit policy preferences and the assessment of negotiable trade permit reforms. European economic review, 1990, 34 : 1399-1426.

Tyers, K., J.K Anderson : Disarray in world food markets : a quantitative assessment. Cambridge, Cambridge University Press, 1992.

Voituriez, T.: What explains price volatility changes in commodity markets? Answers from world palm oil market. Paper presented to the Congress of the International Association of Agricultural Economist, Berlin August 2000.

Williams, C.W., B.A. Isham : Processing industry capacity and the welfare effect of sugar policies. 1999, AJAE $\underline{81}: 424-441$. 
Wong, G., R. Sturgiss and B. Borrell : The economic consequences of international sugar trade reform. ABARE Discussion Paper $N^{\circ}$ 89.7, Canberra, 1989.

\section{ANNEXE : Schéma général de la filière sucre}

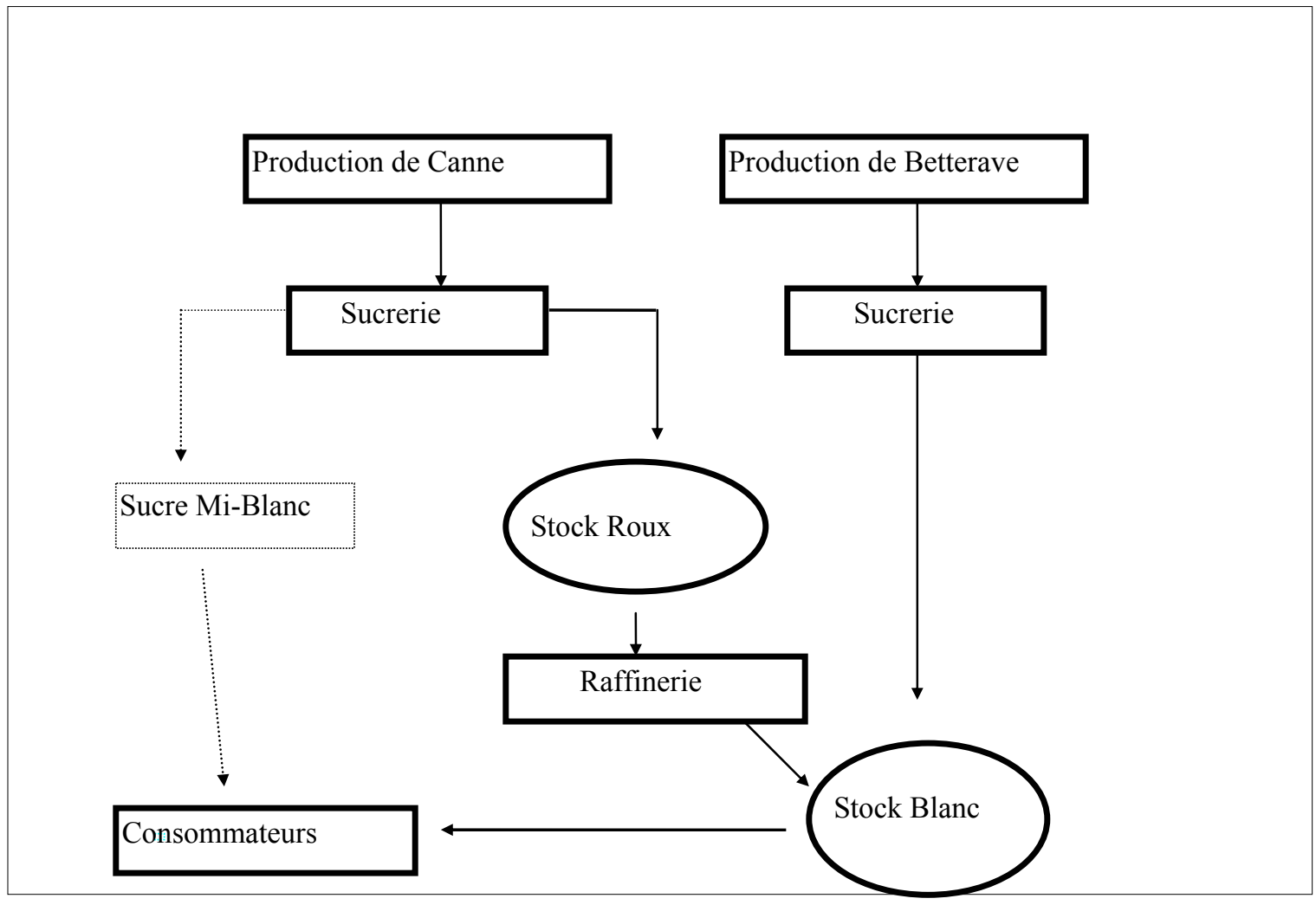

